\title{
Extradural Spinal Cord/Cauda Equina Compression
}

Clinical signs and symptoms suggesting acute or subacute compression of the spinal cord or cauda equina constitute a medical emergency requiring urgent diagnosis in order to effect appropriate therapy aimed at alleviating the pathologic process responsible for the compressive phenomenon. The etiology of the compressive pathology is nonspecific, and runs the gamut of disease-process categories from benign to malignant. The purpose of MR imaging in such cases is to determine the level(s), degree, and, if possible, the type of disease process in order to assist in therapeutic planning gauged toward relieving the neurologic compression (Schnebel et al., 1989; Kent et al., 1992; Jinkins, 1999).

\section{CONVENTIONAL AND FAST SPIN ECHO ACQUISITIONS}

The following sequences comprise the preferred protocol for high-field MR machines. On some machines, alternate gradient-recalled echo acquisitions may be more desirable. In addition, occasionally, intravenous (i.v.) contrast enhanced alternate protocols may be useful to evaluate the integrity of the blood-nerve barrier in certain cases of suspected compressive radiculopathy, or in cases of epidural masses of ambiguous etiology (e.g., epidural neoplasia/infection versus intervertebral disc fragment).

Table A8.7.1 lists the hardware necessary to perform the procedure, along with appropriate parameters. The available gradient strength will depend on the scanner, and the echo times (e.g., $T_{\mathrm{E}}$ ) given in other tables will be varied accordingly (the smaller the gradient strength, the longer the echo time for a particular scan).

The entire protocol should take 35 to 45 min to complete.

NOTE: Be sure that technicians and nurses have immediate access to any emergency equipment that may be relevant to a given study, or that may be needed for a particular patient, such as crash carts or oxygen.

\section{Set up patient and equipment}

1. Interview (screen) the patient to ensure that he or she has no contraindications such as cardiac pacemakers or other implants containing ferromagnetic materials. Also be sure to find out if the patient has any health conditions that may require the presence of special emergency equipment during the scanning procedure, or necessitate any other precautions.

Generally standard screening forms are used for all patients scanned in a magnetic resonance system.

Table A8.7.1 Equipment Parameters for Spine Imaging in Cases of Suspected Extradural Spinal Cord/Cauda Equina Compression

\begin{tabular}{ll}
\hline Coil type & $\begin{array}{l}\text { Cervical, thoracic, lumbar: phase array } \\
\text { surface coil (or other depending upon } \\
\text { machine compatibility and availability) } \\
\text { Flow compensation }\end{array}$ \\
$\begin{array}{l}\text { Any level (optional, if available) } \\
\text { Reripheral gating }\end{array}$ & Thoracic spine only (optional) \\
Use of contrast agents & Thoracic spine only (optional) \\
\hline
\end{tabular}




\begin{tabular}{ll}
\hline Patient position & Supine \\
Scan type & Gradient echo \\
Imaging plane (orientation) & Transverse \\
Slice or volume center & Centered on: \\
& Cervical spine: thyroid cartilage \\
& Thoracic spine: nipple line \\
& Lumbar spine: iliac crests \\
Echo time $\left(T_{\mathrm{E}}\right)$ & As short as possible \\
Repeat time $\left(T_{\mathrm{R}}\right)$ & As short as possible \\
Flip angle $(\mathrm{FA})$ & $15^{\circ}$ \\
Fields of view $\left(\mathrm{FOV}, \mathrm{F}_{\mathrm{x}}, \mathrm{V}_{\mathrm{y}}\right)$ & Cervical: $240 \mathrm{~mm}, 240 \mathrm{~mm}$ \\
& Thoracic: $340 \mathrm{~mm}, 340 \mathrm{~mm}$ \\
Resolution $(\Delta x, \Delta y)$ & Lumbosacral: $280 \mathrm{~mm}, 280 \mathrm{~mm}$ \\
& Cervical: $0.94 \mathrm{~mm}, 0.94 \mathrm{~mm}$ \\
Number of data points collected $\left(N_{\mathrm{x}}, N_{\mathrm{y}}\right)$ & Thoracic: $1.25 \mathrm{~mm}, 1.25 \mathrm{~mm}$ \\
Display matrix $\left(D_{\mathrm{x}}, D_{\mathrm{y}}\right)$ & Lumbosacral: $1.09 \mathrm{~mm}, 1.09 \mathrm{~mm}$ \\
Slice thickness $(\Delta z)$ & 256,256 \\
Number of slices & 5 mm \\
Slice gap & Variable \\
Number of acquisitions $\left(N_{\mathrm{acq}}\right)$ & Not applicable \\
Scan time & 1 \\
& $\sim 10$ sec \\
&
\end{tabular}

The presence of any ferromagnetic metals may be a health hazard to the patient when he or she is inside the magnet, and will also affect the imaging. If in doubt as to the exact composition of the items, it is best to exclude patients with any metal implants; see Shellock (1996) for discussion of what implants may be safely scanned using magnetic resonance.

Patients may be accompanied into the magnet room by a friend or family member, who can sit in the room during the scan and comfort the patient as needed. This companion must be screened as well to ensure the absence of loose metal objects on the body or clothing.

2. If the procedure is a research protocol, have the patient sign any necessary consent form.

3. Have the patient remove all jewelry and change into a gown to eliminate any metal that might be found in clothing.

4. Inform the patient about what will occur during the procedure, what he or she will experience while in the magnet, and how to behave, including the following.

a. If earphones or headphones are used to protect the ears from the loud sounds produced by the gradients, the patient will be asked to wear these, but will be able to communicate with you at any time during the imaging.

b. The patient will be given a safety squeeze-bulb or similar equipment to request assistance at any time (demonstrate how this works).

c. For good results the patient should not talk, and should avoid or minimize swallowing or other movement during each scan-i.e., as long as the banging sounds continue. Between scans, talking and swallowing are allowed in most cases, but should be avoided when comparative positional studies are being performed; the patient will be informed when this is the case.

d. Nevertheless, the patient may call out at any time if he or she feels it necessary.

A8.7.2 
Table A8.7.3 Primary Clinical Imaging Parameters for Sequence 2 ( $T_{1}$-Weighted Image)

\begin{tabular}{|c|c|}
\hline Patient position & Supine \\
\hline Scan type & Conventional spin echo \\
\hline Imaging plane (orientation) & Sagittal \\
\hline Central slice or volume center & $\begin{array}{l}\text { Centered on: } \\
\text { Cervical: 3rd cervical vertebra } \\
\text { Thoracic: 6th thoracic vertebra } \\
\text { Lumbar: 3rd lumbar vertebra }\end{array}$ \\
\hline Echo time $\left(T_{\mathrm{E}}\right)$ & $10 \mathrm{msec}$ \\
\hline Repeat time $\left(T_{\mathrm{R}}\right)$ & $500 \mathrm{msec}$ \\
\hline Flip angle (FA) & $90^{\circ}$ \\
\hline Fields of view $\left(\mathrm{FOV}_{\mathrm{x}}, \mathrm{FOV}_{\mathrm{y}}\right)$ & $\begin{array}{l}\text { Cervical: } 240 \mathrm{~mm}, 240 \mathrm{~mm} \\
\text { Thoracic: } 320 \mathrm{~mm}, 320 \mathrm{~mm} \\
\text { Lumbosacral: } 280 \mathrm{~mm}, 280 \mathrm{~mm} \text { (may } \\
\text { use rectangular field of view [e.g., half } \\
\text { or three-quarter field] if available, or } \\
\text { tailor to region of interest) }\end{array}$ \\
\hline Resolution $(\Delta x, \Delta y)$ & $\begin{array}{l}\text { Cervical: } 0.94 \mathrm{~mm}, 0.94 \mathrm{~mm} \\
\text { Thoracic: } 1.25 \mathrm{~mm}, 1.25 \mathrm{~mm} \\
\text { Lumbosacral: } 1.09 \mathrm{~mm}, 1.09 \mathrm{~mm}\end{array}$ \\
\hline Number of data points collected $\left(N_{\mathrm{x}}, N_{\mathrm{y}}\right)$ & 256,256 \\
\hline Display matrix $\left(D_{\mathrm{x}}, D_{\mathrm{y}}\right)$ & 256,256 \\
\hline Slice thickness $(\Delta z)$ & $\begin{array}{l}\text { Cervical: } 3 \mathrm{~mm} \\
\text { Thoracic: } 3 \mathrm{~mm} \\
\text { Lumbar: } 4 \mathrm{~mm}\end{array}$ \\
\hline Number of slices & $\begin{array}{l}\text { As many as needed to cover the region } \\
\text { of interest }\end{array}$ \\
\hline Slice gap & $\begin{array}{l}\text { Cervical: } 0.5 \mathrm{~mm} \\
\text { Thoracic: } 1 \mathrm{~mm} \\
\text { Lumbar: } 1 \mathrm{~mm}\end{array}$ \\
\hline Number of acquisitions $\left(N_{\mathrm{acq}}\right)$ & 4 \\
\hline Flow compensation & Yes (if available) \\
\hline Saturation pulses & $\begin{array}{l}\text { Yes; anterior cervical/thoracic/lumbar } \\
\text { slab to saturate larynx/vessels/heart }\end{array}$ \\
\hline Slice series & $\begin{array}{l}\text { Left to right or the reverse depending } \\
\text { on preference }\end{array}$ \\
\hline Scan time & $\sim 8 \min$ \\
\hline
\end{tabular}

5. Have the patient mount onto the table. Either before or right after the patient lies down, set up any triggering devices or other monitoring equipment that is to be used.

6. Center the coil over the region where the key information is desired.

Make sure that the body is constrained to prevent motion, especially if high-resolution scans are to be run.

7. If needed, place a pillow or other support under the knees to make the patient more comfortable.

8. Use the centering light to position the patient (cervical spine: thyroid cartilage; thoracic spine: nipple line; lumbar spine: iliac crests) and put him or her into the center of the magnet.

Once this step has been performed, so long as the patient does not move on the table, the table itself can be moved and then replaced in the same position as before without jeopardizing the positioning of one scan relative to another.

Extradural Spine

A8.7.3

Supplement 5 
Table A8.7.4 Primary Clinical Imaging Parameters for Sequence 3 ( $T_{2}$-Weighted Image, Fast Spin Echo)

\begin{tabular}{|c|c|}
\hline Patient position & Supine \\
\hline Scan type & Fast spin echo \\
\hline Imaging plane (orientation) & Sagittal \\
\hline Slice or volume center & $\begin{array}{l}\text { Centered on area of interest (as in } \\
\text { sequence 2, Table A8.7.3) }\end{array}$ \\
\hline Echo time $\left(T_{\mathrm{E}}\right)$ & $100 \mathrm{msec}$ \\
\hline Echo train length (ETL) & 8 \\
\hline Repeat time $\left(T_{\mathrm{R}}\right)$ & $4000 \mathrm{msec}$ \\
\hline Flip angle (FA) & $90^{\circ}$ \\
\hline Fields of view $\left(\mathrm{FOV}_{\mathrm{x}}, \mathrm{FOV}_{\mathrm{y}}\right)$ & As in sequence 2, Table A8.7.3 \\
\hline Resolution $(\Delta x, \Delta y)$ & $\begin{array}{l}\text { Cervical: } 0.47 \mathrm{~mm}, 0.47 \mathrm{~mm} \\
\text { Thoracic: } 0.63 \mathrm{~mm}, 0.63 \mathrm{~mm} \\
\text { Lumbosacral: } 0.55 \mathrm{~mm}, 0.55 \mathrm{~mm}\end{array}$ \\
\hline Number of data points collected $\left(N_{\mathrm{x}}, N_{\mathrm{y}}\right)$ & 512,512 \\
\hline Display matrix $\left(D_{\mathrm{x}}, D_{\mathrm{y}}\right)$ & 512,512 \\
\hline Slice thickness $(\Delta z)$ & $\begin{array}{l}\text { Cervical: } 3 \mathrm{~mm} \\
\text { Thoracic: } 3 \mathrm{~mm} \\
\text { Lumbar: } 4 \mathrm{~mm}\end{array}$ \\
\hline Number of slices & Varies with spinal level \\
\hline Slice gap & $\begin{array}{l}\text { Cervical: } 0.5 \mathrm{~mm} \\
\text { Thoracic: } 1 \mathrm{~mm} \\
\text { Lumbar: } 1 \mathrm{~mm}\end{array}$ \\
\hline Number of acquisitions $\left(N_{\text {acq }}\right)$ & 1 \\
\hline Flow compensation & Yes (if available) \\
\hline Saturation pulses & $\begin{array}{l}\text { Yes; anterior cervical/thoracic/lumbar } \\
\text { slabs to saturate larynx/vessels/heart }\end{array}$ \\
\hline Fat suppression & Yes \\
\hline Slice series & $\begin{array}{l}\text { Left to right or the reverse depending } \\
\text { on preference }\end{array}$ \\
\hline Scan time & $\sim 4 \min$ \\
\hline
\end{tabular}

9. If the patient is unable to hold still, provide an appropriate sedative.

\section{Sequence 1: Rapid positioning pilot}

10. To validate the patient's position, run the system's pilot (or scout) scan (sequence 1) to ensure correct location of the neck in three dimensions, using the imaging sequence given in Table A8.7.2 or similar parameters.

This sequence usually consists of three orthogonal planes to allow subsequent localization. The images are often also used later to determine where to place the saturation pulses and to set up total coverage of the volume of interest.

\section{Sequence 2: Sagittal $T_{1}$-weighted conventional spin echo}

11. Set the imaging parameters as shown in Table A8.7.3.

12. Use the pilot image to locate the spine in three dimensions to ensure coverage of the region of interest (e.g., cervical, thoracic, or lumbosacral spine).

13. Let the patient know you are ready and begin the scan. 
Table A8.7.5 Primary Clinical Imaging Parameters for Sequence 4 ( $T_{1}$-Weighted Images)

\begin{tabular}{|c|c|}
\hline Patient position & Supine \\
\hline Scan type & Conventional spin echo \\
\hline Imaging plane (orientation) & Transverse \\
\hline Central slice or volume center & $\begin{array}{l}\text { Centered on the area of interest (as in } \\
\text { sequence 2, Table A8.7.3) }\end{array}$ \\
\hline Echo time $\left(T_{\mathrm{E}}\right)$ & $10 \mathrm{msec}$ \\
\hline Repeat time $\left(T_{\mathrm{R}}\right)$ & $500 \mathrm{msec}$ \\
\hline Flip angle (FA) & $90^{\circ}$ \\
\hline Fields of view $\left(\mathrm{FOV}_{\mathrm{x}}, \mathrm{FOV}_{\mathrm{y}}\right)$ & As in sequence 2, Table A8.7.3 \\
\hline Resolution $(\Delta x, \Delta y)$ & $\begin{array}{l}\text { Cervical: } 0.94 \mathrm{~mm}, 0.94 \mathrm{~mm} \\
\text { Thoracic: } 1.25 \mathrm{~mm}, 1.25 \mathrm{~mm} \\
\text { Lumbosacral: } 1.09 \mathrm{~mm}, 1.09 \mathrm{~mm}\end{array}$ \\
\hline Number of data points collected $\left(N_{\mathrm{x}}, N_{\mathrm{y}}\right)$ & 256,256 \\
\hline Display matrix $\left(D_{\mathrm{x}}, D_{\mathrm{y}}\right)$ & 256,256 \\
\hline Slice thickness $(\Delta z)$ & $\begin{array}{l}\text { Cervical: } 3 \mathrm{~mm} \\
\text { Thoracic: } 3-8 \mathrm{~mm} \\
\text { Lumbar: } 4 \mathrm{~mm}\end{array}$ \\
\hline Number of slices & Varies with spinal level \\
\hline Slice gap & $\begin{array}{l}\text { Cervical: } 1 \mathrm{~mm} \\
\text { Thoracic: } 1-2 \mathrm{~mm} \\
\text { Lumbar: } 1 \mathrm{~mm}\end{array}$ \\
\hline Number of acquisitions $\left(N_{\text {acq }}\right)$ & 2 \\
\hline Slice location & See text (Basic Protocol, step 16) \\
\hline Saturation pulses & No \\
\hline Scan time & $\sim 4 \mathrm{~min}$ \\
\hline
\end{tabular}

\section{Sequence 3: Sagittal $T_{2}$-weighted fast spin echo, fat suppressed}

14. Review the pilot scans and ensure that the saturation pulse is correctly placed anterior to above the slab of interest.

15. Run sequence 3 according to Table A8.7.4.

\section{Sequence 4: Transverse $T_{1}$-weighted conventional spin echo}

16. Using the midline sagittal $T_{1}$-weighted image acquired in sequence 2 , set the transverse acquisition parameters as follows:

a. Cervical spine: stacked images from $\mathrm{C} 1$ through C7-T1.

b. Thoracic spine: stacked images through levels of interest.

c. Lumbosacral spine: 5 slices each, angled to the plane of the intervertebral disc at L3-4, L4-5, and L5-S1; one slice each, angled to the intervertebral disc at L1-2 and $\mathrm{L} 2-3$.

17. Supplement additional slices according to visible disease present or to clinical query.

18. Run the sequence according to Table A8.7.5.

\section{Sequence 5: Transverse $T_{2}$-weighted fast spin echo}

19. Using the midline $T_{1}$-weighted image acquired in sequence 2 , repeat the setup as in Table A8.7.6.

20. Run sequence 5 according to Table A8.7.6.

Extradural Spine

A8.7.5 
ALTERNATE PROTOCOL 1

ALTERNATE PROTOCOL 2

\section{Extradural Spinal Cord/Cauda Equina Compression}

A8.7.6
Table A8.7.6 Primary Clinical Imaging Parameters for Sequence 5 ( $T_{2}$-Weighted Image, Fast Spin Echo)

\begin{tabular}{|c|c|}
\hline Patient position & Supine \\
\hline Scan type & Fast spin echo \\
\hline Imaging plane (orientation) & Transverse \\
\hline Central slice or volume center & $\begin{array}{l}\text { Centered on the region of interest (as } \\
\text { in sequence } 2 \text {, Table A8.7.3) }\end{array}$ \\
\hline Echo time $\left(T_{\mathrm{E}}\right)$ & $100 \mathrm{msec}$ \\
\hline Echo train length (ETL) & 8 \\
\hline Repeat time $\left(T_{\mathrm{R}}\right)$ & $4000 \mathrm{msec}$ \\
\hline Flip angle (FA) & $90^{\circ}$ \\
\hline Fields of view $\left(\mathrm{FOV}_{\mathrm{x}}, \mathrm{FOV}_{\mathrm{y}}\right)$ & As in sequence 2, Table A8.7.3 \\
\hline Resolution $(\Delta x, \Delta y)$ & $\begin{array}{l}\text { Cervical: } 0.94 \mathrm{~mm}, 0.94 \mathrm{~mm} \\
\text { Thoracic: } 1.25 \mathrm{~mm}, 1.25 \mathrm{~mm} \\
\text { Lumbosacral: } 1.09 \mathrm{~mm}, 1.09 \mathrm{~mm}\end{array}$ \\
\hline Number of data points collected $\left(N_{\mathrm{x}}, N_{\mathrm{y}}\right)$ & 256,256 \\
\hline Display matrix $\left(D_{\mathrm{x}}, D_{\mathrm{y}}\right)$ & 256,256 \\
\hline Slice thickness $(\Delta z)$ & $\begin{array}{l}\text { Cervical: } 3 \mathrm{~mm} \\
\text { Thoracic: } 3-8 \mathrm{~mm} \\
\text { Lumbar: } 4 \mathrm{~mm}\end{array}$ \\
\hline Number of slices & Varies with spinal level \\
\hline Slice gap & $\begin{array}{l}\text { Cervical: } 1 \mathrm{~mm} \\
\text { Thoracic: } 1-2 \mathrm{~mm} \\
\text { Lumbar: } 1 \mathrm{~mm}\end{array}$ \\
\hline Number of acquisitions $\left(N_{\text {acq }}\right)$ & 2 \\
\hline Slice locations & See Basic Protocol, step 16 \\
\hline Saturation pulses & No \\
\hline Scan time & $\sim 4 \min$ \\
\hline
\end{tabular}

\section{GRADIENT-RECALLED ECHO ACQUISITIONS}

With some machines, or according to preferences, gradient-recalled echo acquisitions may be used in the sagittal and/or transverse planes to clearly distinguish between the spinal cord and the extradural tissue comprising this structure. This is especially true in cases where the epidural mass consists of acute blood products (e.g., deoxyhemoglobin) that are paramagnetic and are better characterized by $T_{2}{ }^{*}$ gradient-recalled echo images than by $T_{2}$ fast spin echo acquisitions.

\section{Sequence 6: Sagittal gradient-recalled echo}

1. Run the sequence according to Table A8.7.7.

\section{Sequence 7: Transverse gradient-recalled echo}

2. Run the sequence according to Table A8.7.8.

\section{CONTRAST ENHANCED ACQUISITIONS}

In some situations (e.g., infection, neoplasia) i.v. paramagnetic contrast material may be indicated.

\section{Materials}

Normal saline $(0.9 \% \mathrm{NaCl})$, sterile

Intravenous MRI contrast agent (e.g., Magnevist, Omniscan, or Prohance) 
Table A8.7.7 Primary Clinical Imaging Parameters for Sequence $6\left(T_{2}{ }^{*}\right.$ Gradient Recalled Echo)

\begin{tabular}{ll}
\hline Patient position & Supine \\
Scan type & 2-D gradient echo \\
Imaging plane (orientation) & Sagittal \\
Central slice or volume center & Centered on the region of interest (as \\
& in sequence 2, Table A8.7.3) \\
Echo time $\left(T_{\mathrm{E}}\right)$ & $15 \mathrm{msec}$ \\
Repeat time $\left(T_{\mathrm{R}}\right)$ & $500 \mathrm{msec}$ \\
Flip angle $(\mathrm{FA})$ & $10^{\circ}$ to $20^{\circ}$ \\
Fields of view $\left(\mathrm{FOV}_{\mathrm{x}}, \mathrm{FOV}_{\mathrm{y}}\right)$ & As in sequence 2, Table A8.7.3 \\
Resolution $(\Delta x, \Delta y)$ & Cervical: $0.94 \mathrm{~mm}, 0.94 \mathrm{~mm}$ \\
& Thoracic: $1.25 \mathrm{~mm}, 1.25 \mathrm{~mm}$ \\
& Lumbosacral: $1.09 \mathrm{~mm}, 1.09 \mathrm{~mm}$ \\
Number of data points collected $\left(N_{\mathrm{x}}, N_{\mathrm{y}}\right)$ & 256,256 \\
Display matrix $\left(D_{\mathrm{x}}, D_{\mathrm{y}}\right)$ & 256,256 \\
Slice thickness $(\Delta z)$ & $3 \mathrm{~mm}$ \\
Number of slices & Varies with spinal level \\
Slice gap & $\leq 1 \mathrm{~mm}$ \\
Number of acquisitions $\left(N_{\mathrm{acq}}\right)$ & 3 \\
Slice location & See Basic Protocol, step 16 \\
Flow compensation & Yes (if available) \\
Saturation pulses & Yes \\
Scan time & 6 min \\
\hline
\end{tabular}

Table A8.7.8 Primary Clinical Imaging Parameters for Sequence $7\left(T_{2}{ }^{*}\right.$ Gradient Recalled Echo)

\begin{tabular}{|c|c|}
\hline Patient position & Supine \\
\hline Scan type & Gradient echo \\
\hline Imaging plane (orientation) & Transverse \\
\hline Central slice or volume center & $\begin{array}{l}\text { Centered on the region of interest (as } \\
\text { in sequence } 2 \text {, Table A8.7.3) }\end{array}$ \\
\hline Echo time $\left(T_{\mathrm{E}}\right)$ & $15 \mathrm{msec}$ \\
\hline Repeat time $\left(T_{\mathrm{R}}\right)$ & $500 \mathrm{msec}$ \\
\hline Flip angle (FA) & $10^{\circ}$ to $20^{\circ}$ \\
\hline Fields of view $\left(\mathrm{FOV}_{\mathrm{x}}, \mathrm{FOV}_{\mathrm{y}}\right)$ & As in sequence 2, Table A8.7.3 \\
\hline \multirow[t]{3}{*}{ Resolution $(\Delta x, \Delta y)$} & Cervical: $0.94 \mathrm{~mm}, 0.94 \mathrm{~mm}$ \\
\hline & Thoracic: $1.25 \mathrm{~mm}, 1.25 \mathrm{~mm}$ \\
\hline & Lumbosacral: $1.09 \mathrm{~mm}, 1.09 \mathrm{~mm}$ \\
\hline Number of data points collected $\left(N_{\mathrm{x}}, N_{\mathrm{y}}\right)$ & 256,256 \\
\hline Display matrix $\left(D_{\mathrm{x}}, D_{\mathrm{y}}\right)$ & 256,256 \\
\hline Slice thickness $(\Delta z)$ & $3-4 \mathrm{~mm}$ \\
\hline Number of slices & Varies with spinal level \\
\hline Slice gap & $\leq 1 \mathrm{~mm}$ \\
\hline Number of acquisitions $\left(N_{\mathrm{acq}}\right)$ & 1 \\
\hline Slice location & See Basic Protocol, step 16 \\
\hline Flow compensation & Yes (if available) \\
\hline Saturation pulses & Yes \\
\hline Scan time & $\sim 2 \min$ \\
\hline
\end{tabular}


1. Use the same equipment and perform patient setup as in Basic Protocol, steps 1 to 4.

2. Establish an i.v. line from which the contrast agent can be injected, and attach this line securely to the patient so that movement into or out of the magnet will not pull at the patient's arm. Resume steps 5 to 9 in the Basic Protocol.

It is preferable to insert the line prior to imaging and to leave the patient in the magnet, with no intervening motion, between the scans run before contrast agent injection and those run after injection.

\section{Scan pilot}

3. Run a rapid three-plane positioning pilot scan (see Basic Protocol, sequence 1).

\section{Sequences 8 and 9: $i . v$. contrast enhanced $T_{1}$-weighted spin echo}

4. Leaving the patient in the magnet, inject the contrast agent, flush the line with $10 \mathrm{ml}$ saline, and then immediately run sagittal (sequence 8) and transverse (sequence 9) $T_{1}$-weighted image sequences (see Basic Protocol, sequences 2 and 4, Tables A8.7.3 and A8.7.5; sequences 8 and 9 are the same sequences 2 and 4, respectively, but with the contrast agent injected into the patient).

An i.v. dose of $0.1 \mathrm{mmol} / \mathrm{kg}$ of contrast agent is usually given.

\section{COMMENTARY}

\section{Background Information}

Acute or subacute compression of the spinal cord in the cervical or thoracic spinal cord, or of the cauda equina in the thoracolumbar region, is a major neurologic emergency almost always requiring urgent medical attention in order to minimize permanent neural deficit. The causes of such compression are nonspecific and can be degenerative, traumatic, inflammatory, or neoplastic, or may constitute acquired conditions of a different nature than the pathologic entities mentioned (Fig. A8.7.1).
Extradural Spinal
Cord/Cauda
Equina
Compression

A8.7.8
A

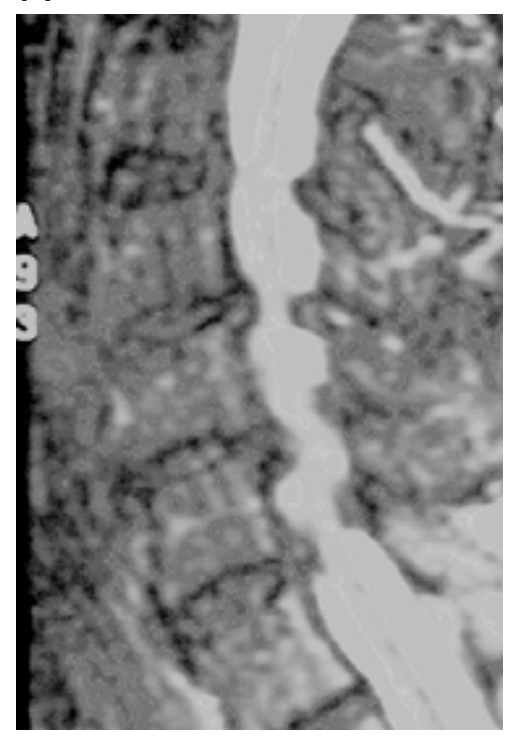

$\mathrm{B}$

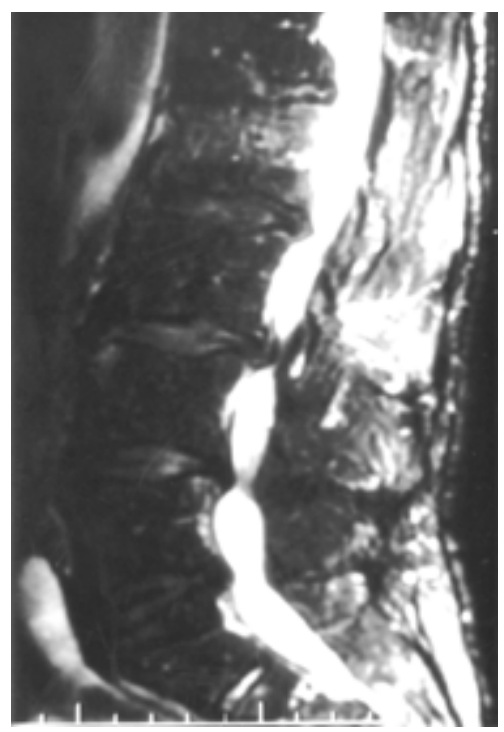

Figure A8.7.1 Benign multilevel extradural spinal cord/cauda equina compression. (A) Sagittal $T_{2}$-weighted $\left(T_{\mathrm{R}}=4000 \mathrm{msec}, T_{\mathrm{E}}=100 \mathrm{msec}\right)$ fat suppressed image shows multilevel spinal cord compression in the cervical region due to generalized spondylosis. (B) Sagittal $T_{2}$-weighted $\left(T_{\mathrm{R}}=\right.$ $4000 \mathrm{msec}, T_{\mathrm{E}}=100 \mathrm{msec}$ ) fat suppressed image shows multilevel degenerative cauda equina compression in the lumbosacral region also secondary to generalized spondylosis. 
Regardless of etiology, MR imaging, an almost imperative element, is the acute or even emergent evaluation of these patients in order to analyze the degree, level(s), and extent of spinal cord/cauda equina compression. In addition, MR imaging may enable a listing of the narrowest possible differential diagnostic possibility(ies). This will materially aid, in the most timely fashion, the therapeutic planning of such acutely neurologically debilitated patients.

\section{Clinical Parameters and Troubleshooting}

Cerbrospinal fluid (CSF) flow, cardiac, laryngeal, body wall, and other sources of motion can produce artifacts that can on occasion significantly degrade the images. Proper spatial (e.g., prevertebral) saturation pulses and sometimes flow compensation pulses and/or cardiac/respiratory gating can reduce these artifacts significantly. In many instances these artifacts may be difficult or impossible to overcome from patient to patient.

\section{Anticipated Results}

The goal of starting the patient with clinically suspected spinal cord or cauda equina compression is to pinpoint the level(s), degrees, and extent of disease in order to significantly aid in the urgent treatment planning in these patients in an effort to minimize permanent neurologic deficit and enhance timely clinical patient outcome.

\section{Literature Cited}

Jinkins, J.R. 1999. MR evaluation of stenosis involving the neural foramina lateral recesses and central canal of the lumbosacral spine. Neuroimag. Clin. North Am. 7:493-511.

Kent, D.L., Haynor, D.R., Larson, E.B., and Deyo, R.A. 1992. Diagnosis of lumbar spinal stenosis in adults: A metaanalysis of the accuracy of CT, MR, and myelgraphy. Am. J. Roentgenol. 158:1135-1144.

Schnebel, B., Kingston, S., Watkins, R., and Dillon, W. 1989. Comparison of MRI to contrast CT in the diagnosis of spinal stenosis. Spine 14:332337.

Shellock, F.G. 1996. Pocket Guide to MR Procedures and Metallic Objects. Lippincott-Raven, Philadelphia.

Contributed by J. Randy Jinkins and David D. Stark

Downstate Medical Center

State University of New York

Brooklyn, New York
Extradural Spine

A8.7.9 